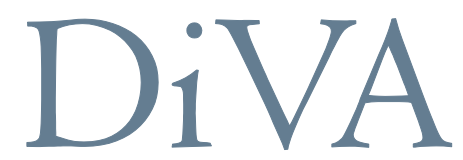

http://uu.diva-portal.org

This is an author's version of a paper presented at Academic MindTrek 2012 International Conference on Media of the Future, October 03-05, 2012, Tampere, Finland.

Citation for the published paper:

Zabramski, Stanislaw, Stuerzlinger, Wolfgang

"The Effect of Shape Properties on Ad-hoc Shape Replication with Mouse, Pen, and Touch Input"

MindTrek '12 Proceeding of the 16th International Academic MindTrek Conference, 2012, pp. 275-278

ISBN: 978-1-4503-1637-8

http://dx.doi.org/10.1145/2393132.2393192

Access to the published version may require subscription. 


\section{The Effect of Shape Properties on Ad-hoc Shape Replication with Mouse, Pen, and Touch Input}

\author{
Stanislaw Zabramski \\ Informatics and Media \\ Uppsala University \\ Uppsala, Sweden \\ stanislaw.zabramski@im.uu.se
}

\author{
Wolfgang Stuerzlinger \\ Computer Science and Engineering \\ York University \\ Toronto, Canada \\ www.cse.yorku.ca/ wolfgang
}

\begin{abstract}
This paper summarizes observations from four empirical studies focusing on shape replication with three input methods. The aim was to identify and assess how the components of several semirandomly generated shapes influence how accurately untrained users can replicate each of these components. We found that the pen is the least and touch the most error-prone method when used for drawing. Additionally, the distribution of errors was analyzed. The results may be used to predict which shape properties make shape replication more difficult. Additionally, the results may be used to design shapes that are easy to replicate.
\end{abstract}

\section{Categories and Subject Descriptors}

H.5.2 User Interfaces: Input devices and strategies.

\section{General Terms}

Measurement, Performance, Experimentation, Human Factors.

\section{Keywords}

Mouse, stylus, pen, touch, shape, replication, tracing, drawing.

\section{INTRODUCTION}

How accurately humans can interact with a system depends not only on the input device but also on task difficulty. Traditional research on computer input methods focuses on the performance aspects of navigational pointing or selection tasks. However, it is not possible to directly apply existing HCI models, such as Fitts' Law or the Law of Steering, to predict more complex tasks, such as replicating a drawing. One issue is that these models are spatiotemporally restrictive, as they cannot model systematic deviations from the classical speed-accuracy trade-off. For unconstrained free-hand line-tracing, as typically used in creative drawing or gesturing, the outcome depends also on the tool used as well as what shape and how accurately it is being drawn. A classic example is the problem of adding a signature to a document with a mouse as input device.

Free-hand drawing with computer systems dates back to Sutherland's Sketchpad in the early sixties [1]. However, at that time the term sketching was used for what is now known as

Permission to make digital or hard copies of all or part of this work for personal or classroom use is granted without fee provided that copies are not made or distributed for profit or commercial advantage and that copies bear this notice and the full citation on the first page. To copy otherwise, or republish, to post on servers or to redistribute to lists, requires prior specific permission and/or a fee.

MindTrek 2012, October 3-5, 2012, Tampere, FINLAND.
Computer-Aided Design. The research that followed focused on engineering or architecture. Consequently, systems supported the drawing process by constraining the user's freedom to prevent errors, instead of enabling free-hand drawing. Yet, free-hand drawing provides the opportunity to create a shape that is not predefined and which can be dynamically reshaped during the tracing process. That process will in general be influenced by the properties of the tool used for drawing.

\section{BACKGROUND AND RELATED WORK}

Each different popular input device, such as a mouse, stylus, or touch sensitive screen, delivers a different user experience that potentially translates to different outcomes for the same action. In contrast to pure pointing tasks - where the main user's goal is getting from point $\mathrm{A}$ to point $\mathrm{B}$ as fast as possible - shape replication demands a different context. To describe such a continuous user action, e.g. performed on a touch-sensitive surface, we can use a time-series of $2 \mathrm{D}$ coordinates of the center of the touch area - the Contact Area Model [2] - and consider it as an example of line tracing.

Sketching behavior has been already experimentally studied [3], [4] but mainly in collaborative and creative contexts. Thus there is a need to perform dedicated and comparative user studies to expand our knowledge how the computer input methods influence an individual's accuracy in shape replication tasks.

\section{METHODS AND PROCEDURES}

We performed a series of experiments to compare the mouse, pen, and touch-input in a shape replication task. We measured the timing and deviation from the ideal shape in a tracing task with or without visual feedback. We used several semi-randomly generated shapes that contain the most common features of geometrical shapes.

\subsection{Shapes}

The geometrical properties of shapes have an extensive impact on human visual perception [5]. For our studies, three asymmetrical, semi-random, non-meaningful, contour shapes were generated based on an existing method, Method 4 of [6]. This method describes a procedure for making wholly or partially curved shapes from the angular shapes consisting of straight line segments. It may be decided arbitrarily or randomly which angles are to be replaced with curved elements or left as originally drawn. We modified this method to generate shapes that consist of at least two instances of each of the following elements: convex corner, concave corner, straight segment, and curved segment. The parameters, such as length or angles, were randomized under the constraint that the segments do not intersect at any point. Shapes were automatically closed via a straight segment 
connecting the last generated element with the beginning of the first one. To remove potential bias, shapes were selected not to resemble letters, well-known shapes, or popular objects.

\subsection{Feedback during tracing}

Previous research identified that visual feedback is an external aid in drawing tasks. This is supported by the distributed cognition view of cognitive support [7]. Visual feedback can also be seen as a trigger of difference-sensing and difference-reducing feedback mechanisms in the human mind [8]. Based on experimental results on interaction feedback [9], this might lead one to expect a positive influence of visual feedback. However, this hypothesis was not confirmed in two previous studies [10], [11]. Therefore we consider these two conditions as equal.

\subsection{Average Deviation (Error)}

Error is here a measure of the deviation between the original shape and the user input. It is equal to the average value of pixelwise distances between a number of corresponding points located on the sampled version of original shape and on user-generated one, see [11].

\section{EXPERIMENTAL STUDIES}

A series of four comparative experimental studies on shape replication with mouse, pen and touch input were performed. After a short introductory session in MS Paint, greyed-out shapes were displayed (e.g. Fig.2-left, shown full screen with $70 \%$ opacity). Participants were instructed to trace over the shape clockwise, in one stroke, starting from the top (right) corner. They used every input method once with or without visual feedback of their input. Visual feedback took the form of a black line of the same thickness as the presented shape. Input methods were randomly assigned to counter potential order effects. Task time was not restricted but we recorded timing information for every part of the stroke and stored each participant's final drawing as a bitmap. We used a HP Touchsmart TM2-1090eo Tablet PC with a 12.1 inch diagonal LED display and a resolution of $1280 * 800$ pixels, equipped with a pen and touch sensitive display, as well as a Logitech basic optical mouse. The PC was used in "tablet mode" with the pen and finger input and in "laptop mode" for the mouse. All three input methods used default settings and standard Windows 7 system cursors were visible while interacting. All the studies followed similar scenarios but varied in terms of feedback and shapes (see Table 1). Study 1 consisted of two parts: a) tracing shape 1 in three sequential speed conditions ( $\mathrm{N}-$ normal, A-accurate, and F-fast) as well as b) tracing shape 2 with transparent ink. Studies 2 and 3 were published before as [10], [11], but we analyze the data from these experiments in a different way here.

Table 1. Details of experiments

\begin{tabular}{|c|c|c|c|}
\hline Study & $\begin{array}{c}\text { Number of } \\
\text { participants }\end{array}$ & Shape & $\begin{array}{c}\text { Feedback } \\
\text { during tracing }\end{array}$ \\
\hline $1 \mathrm{a}$ & 8 & 1 & on \\
\hline $1 \mathrm{~b}$ & 8 & 2 & off \\
\hline 2 & 34 & 2 & on and off \\
\hline 3 & 16 & 1 & on and off \\
\hline 4 & 9 & 3 & on \\
\hline
\end{tabular}

\section{RESULTS}

Similar differences between input devices were found across all four studies, e.g. [11]. They confirm the classic speed-accuracy trade-off with the overall slowest device, the mouse. Touch was consistently the fastest alternative. Touch was the least and pen the most accurate input device. The lack of any temporal or spatial constraint caused a large spread of measured values, depending on subjective operational biases towards speed or accuracy. Touch had the largest amount of error, yet with small variability and very consistent deviations. The presence of visual feedback did not influence the accuracy of tracing as the average deviation was not significantly different across studies.

\subsection{Shape properties and error}

Corners, i.e. elements where two line elements meet at an angle, have been recognized by previous research as perceptually challenging [12]. Also, the time taken to complete a trajectorybased task is increased by the mere presence of a corner [13]. Pastel has shown that because of biomechanical reasons, $45^{\circ}$ corners are easier to negotiate than $90^{\circ}$ or even $135^{\circ}$ corners.

As we generated our shapes semi-randomly, we classify corners into categories not only based on their relation to the original shape (concave, convex), but also based on their angle (acute between $0^{\circ}$ and $90^{\circ}$ and obtuse between $90^{\circ}$ and $180^{\circ}$ ), as well as the context of the types of segments around the corner (line-line, line-curve, curve-line, curve-curve). The corners that were used as starting/ending points of the tracing task were excluded from this analysis because they were not the result of continuous action and the user generated lines may not even meet at that corner. The distributions of users' error at all sampled points were analyzed in relation to the mean value (e.g. Fig.1). Table 2 summarizes the results of this analysis for all three shapes for all input methods categorized by the shape's properties.

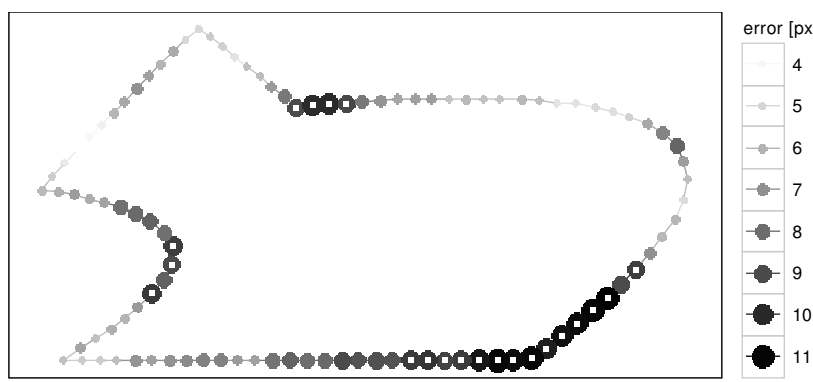

Figure 1. Plot of the average error measured at each sampled point of shape 3 replicated with pen. White squares mark points where the value exceeds the shape's mean + 1SD.

Pastel [13] observed interesting strategies when users were navigating corners: "cutting off the corner" - producing a rounded corner without slowing the pace of movement or "stop and go" producing a sharp corner through temporary deceleration. A similar analysis was performed here. Additionally, we analyzed if these strategies were applied equally to convex and concave corners. Thus and if at least one corner of a given type had been negotiated with the "cutting off" strategy, the whole trial was marked as positive. Table 3 summarizes the results of this analysis in relation to the number of trials for each experiment and for all three shapes. Additionally, we verified if a particular user used a given strategy consistently for at least two of the three input methods. This might indicate a general tendency towards a particular approach. 

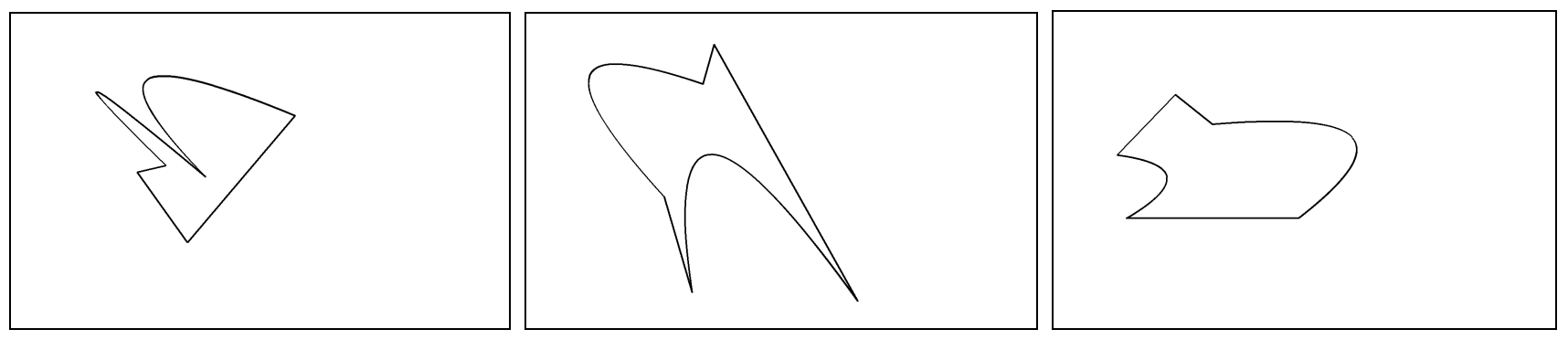

Figure 2. Shapes 1, 2, and 3 (respectively) with their placement and size proportional to the whole computer screen.

Table 2. Deviations for all shape properties for each input method, where: " $\square$ " denotes big user error (values around and bigger than the mean + 1SD); " $\Delta$ " average user error (values around the mean); " $\square$ " small user error (values around and smaller than the mean - 1SD); and " - " property missing in a given shape.

\begin{tabular}{|c|c|c|c|c|c|c|c|c|c|c|c|c|c|c|}
\hline \multirow{2}{*}{\multicolumn{3}{|c|}{ Shape's properties }} & \multicolumn{4}{|c|}{ Shape 1} & \multicolumn{4}{|c|}{ Shape 2} & \multicolumn{4}{|c|}{ Shape 3} \\
\hline & & & \multirow{2}{*}{$\frac{\text { mouse }}{\boldsymbol{\Lambda}}$} & \multirow{2}{*}{$\frac{\text { pen }}{\Delta}$} & \multirow{2}{*}{$\begin{array}{c}\text { touch } \\
\square\end{array}$} & \multirow{2}{*}{$\frac{\text { all }}{4}$} & \multirow{2}{*}{$\frac{\text { mouse }}{\boldsymbol{\Lambda}}$} & \multirow{2}{*}{$\begin{array}{c}\text { pen } \\
\square\end{array}$} & \multirow{2}{*}{$\begin{array}{c}\text { touch } \\
\boldsymbol{\Delta}\end{array}$} & \multirow{2}{*}{$\frac{\text { all }}{4}$} & \multirow{2}{*}{$\begin{array}{c}\text { mouse } \\
\boldsymbol{\Lambda}\end{array}$} & \multirow{2}{*}{$\frac{\text { pen }}{\mathbf{\square}}$} & \multirow{2}{*}{$\begin{array}{c}\text { touch } \\
\square\end{array}$} & \multirow{2}{*}{$\frac{\text { all }}{4}$} \\
\hline \multirow{8}{*}{ lines } & . & long & & & & & & & & & & & & \\
\hline & sudigmt & short & $\square$ & 4 & $\square$ & $\Delta$ & 4 & 4 & $\square$ & 4 & 4 & 4 & 4 & $\boldsymbol{\Delta}$ \\
\hline & \multirow{6}{*}{ curved } & sharp & $\square$ & $\mathbf{\square}$ & $\square$ & $\Delta$ & - & - & - & - & - & - & - & - \\
\hline & & smooth & $\square$ & $\boldsymbol{\square}$ & $\square$ & 4 & $\boldsymbol{4}$ & $\mathbf{a}$ & $\mathbf{\square}$ & $\mathbf{\square}$ & $\mathbf{\square}$ & $\mathbf{\square}$ & 4 & $\boldsymbol{\square}$ \\
\hline & & short & - & - & - & - & - & - & - & - & $\bar{\square}$ & $\mathbf{\square}$ & $\square$ & $\mathbf{\square}$ \\
\hline & & long & $\square$ & $\mathbf{\square}$ & 4 & $\mathbf{\square}$ & 4 & $\boldsymbol{\square}$ & $\square$ & $\mathbf{\square}$ & $\Delta$ & $\mathbf{\square}$ & $\Delta$ & $\Delta$ \\
\hline & & concave & $\mathbf{\square}$ & $\mathbf{\square}$ & $\square$ & $\Lambda$ & 4 & $\mathbf{\square}$ & 4 & $\Delta$ & $\mathbf{\square}$ & $\mathbf{\square}$ & $\square$ & $\Delta$ \\
\hline & & convex & - & - & - & - & 4 & $\square$ & $\square$ & 4 & 4 & $\square$ & 4 & 4 \\
\hline \multirow{8}{*}{ corners } & \multirow{2}{*}{ concave } & acute & 4 & 4 & $\square$ & 4 & 4 & $\square$ & 4 & 4 & - & - & - & - \\
\hline & & obtuse & $\mathbf{\square}$ & 4 & $\square$ & $\Lambda$ & 4 & 4 & $\mathbf{\square}$ & $\Lambda$ & $\mathbf{\square}$ & $\mathbf{\square}$ & $\mathbf{\square}$ & $\mathbf{\square}$ \\
\hline & \multirow{2}{*}{ convex } & acute & $\square$ & $\mathbf{\square}$ & $\square$ & 4 & - & - & - & - & 4 & 4 & $\square$ & 4 \\
\hline & & obtuse & $\mathbf{\square}$ & $\mathbf{\square}$ & $\square$ & $\Delta$ & 4 & $\mathbf{\square}$ & $\mathbf{\square}$ & $\mathbf{\square}$ & $\bar{\square}$ & $\mathbf{\square}$ & 4 & $\mathbf{\square}$ \\
\hline & \multirow{4}{*}{ context } & line-line & - & - & - & - & - & - & - & - & $\square$ & $\square$ & 4 & $\square$ \\
\hline & & line-curve & $\mathbf{\square}$ & $\mathbf{\square}$ & 4 & $\mathbf{\square}$ & 4 & $\mathbf{\square}$ & $\mathbf{\square}$ & $\mathbf{\square}$ & $\mathbf{\square}$ & $\mathbf{\square}$ & $\mathbf{\square}$ & $\mathbf{\square}$ \\
\hline & & curve-line & $\square$ & $\mathbf{\square}$ & $\square$ & $\boldsymbol{4}$ & 4 & $\mathbf{\square}$ & 4 & 4 & $\square$ & $\bar{\square}$ & 4 & $\mathbf{\square}$ \\
\hline & & curve-curve & - & - & - & - & $\boldsymbol{4}$ & $\square$ & [ & $\Delta$ & - & - & - & - \\
\hline
\end{tabular}

Table 3. Percent of corners negotiated with the "cutting off" strategy per input per shape and user consistency.

\begin{tabular}{|c|c|c|c|c|c|c|c|c|c|c|c|c|c|c|c|}
\hline \multirow{2}{*}{$\begin{array}{c}\text { study\# } \\
\& \\
\text { shape }\end{array}$} & \multicolumn{3}{|c|}{ mouse } & \multicolumn{3}{|c|}{ pen } & \multicolumn{3}{|c|}{ touch } & \multicolumn{3}{|c|}{ all inputs } & \multicolumn{3}{|c|}{ consistent strategies } \\
\hline & $\begin{array}{c}\text { convex } \\
{[\%]}\end{array}$ & \begin{tabular}{|c|} 
concave \\
{$[\%]$}
\end{tabular} & $\begin{array}{l}\text { both } \\
{[\%]}\end{array}$ & $\begin{array}{c}\text { convex } \\
{[\%]}\end{array}$ & \begin{tabular}{|c|} 
concave \\
{$[\%]$}
\end{tabular} & $\begin{array}{l}\text { both } \\
{[\%]}\end{array}$ & $\begin{array}{c}\text { convex } \\
{[\%]}\end{array}$ & $\begin{array}{c}\text { concave } \\
{[\%]}\end{array}$ & \begin{tabular}{|l|} 
both \\
{$[\%]$}
\end{tabular} & $\begin{array}{c}\text { convex } \\
{[\%]}\end{array}$ & $\begin{array}{c}\text { concave } \\
{[\%]}\end{array}$ & $\begin{array}{l}\text { both } \\
{[\%]}\end{array}$ & $\begin{array}{c}\text { convex } \\
{[\%]}\end{array}$ & \begin{tabular}{|c|} 
concave \\
{$[\%]$}
\end{tabular} & $\begin{array}{l}\text { both } \\
{[\%]}\end{array}$ \\
\hline 3 & 31 & 6 & 0 & 38 & 13 & 6 & 31 & 13 & 6 & 33 & 10 & 4 & 25 & 6 & 0 \\
\hline 1a N & 50 & 0 & 0 & 50 & 0 & 0 & 75 & 0 & 0 & 58 & 0 & 0 & 75 & 0 & 0 \\
\hline $1 \mathbf{a} \mathbf{A}$ & 63 & 0 & 0 & 38 & 0 & 0 & 25 & 13 & 0 & 42 & 4 & 0 & 38 & 0 & 0 \\
\hline 1a $F$ & 25 & 25 & 13 & 63 & 0 & 0 & 75 & 0 & 0 & 54 & 8 & 4 & 63 & 0 & 0 \\
\hline shape1 & 42 & 8 & 3 & 47 & 3 & 2 & 52 & 6 & 2 & 47 & 6 & 2 & 50 & 2 & 0 \\
\hline $1 \mathrm{~b}$ & 0 & 50 & 0 & 25 & 50 & 25 & 25 & 75 & 25 & 17 & 58 & 17 & 13 & 75 & 13 \\
\hline 2 & 6 & 68 & 6 & 12 & 53 & 3 & 18 & 47 & 12 & 12 & 56 & 7 & 3 & 62 & 3 \\
\hline shape 2 & 3 & 59 & 3 & 18 & 51 & 14 & 21 & 61 & 18 & 14 & 57 & 12 & 8 & 68 & 8 \\
\hline shape3 & 78 & 33 & 22 & 44 & 56 & 11 & 56 & 89 & 44 & 59 & 59 & 26 & 56 & 56 & 0 \\
\hline
\end{tabular}


Table 4. Ratio of average error to shape's length per device.

\begin{tabular}{|c|c|c|c|c|}
\hline Shape & Length $[\mathbf{p x}]$ & Mouse & Pen & Touch \\
\hline 1 & 1601 & $\mathbf{4 . 8 9}-\mathrm{e} 03$ & $\mathbf{4 . 0 1}-\mathrm{e} 03$ & $\mathbf{8 . 0 3}-\mathrm{e} 03$ \\
\hline 2 & 2275 & $\mathbf{4 . 5 0}-\mathrm{e} 03$ & $\mathbf{3 . 6 6}-\mathrm{e} 03$ & $\mathbf{4 . 5 8}-\mathrm{e} 03$ \\
\hline 3 & 1451 & $\mathbf{5 . 0 3}-\mathrm{e} 03$ & $\mathbf{4 . 8 9}-\mathrm{e} 03$ & $\mathbf{8 . 4 5}-\mathrm{e} 03$ \\
\hline
\end{tabular}

\section{DISCUSSION}

We expected the elements of the investigated shapes to have an impact on user error. We distinguish between two kinds of effects: shape-related effects and input-related ones. We believed that errors would increase with segment length (Tab. 4). However, the "longest" shape, \#2, was not the most error-prone. Touch input was different from mouse and pen in terms of performance. Likely due to the fat finger or perceived input point problems [14], shapes are replicated equally badly within each study. However, some shapes are harder to replicate with touch. E.g., shape 2 was harder than shape 1, even though both share the same set of shape properties. Shape 2 seems to be easier to replicate with a mouse than the other two input methods. Yet, acute convex and concave corners seem to pose fewer problems to mouse users, and users consistently used the "cutting off the corner" strategy here. Users cut off convex corners more often for shape 1, while concave corners were cut off more likely for shape 2 . For shape 3 there was no preference, which may be due to the more compact body of it. For pen users, straight lines are also easier to replicate, likely due to better high-accuracy control with this device. We could not clearly identify an influence of the ordering of the elements. Only the curve to line transition was noticeably easier to replicate with touch.

\section{CONCLUSION}

The precise line-tracing task used here is related to tasks ranging from free-hand drawing and design to complex lasso or linear selections. Our studies found that user's accuracy in these tasks is affected in various ways by the input method and the properties of the drawn shape. Pen is the least and touch is the most error-prone input method when used for tracing.

The results can be used in two ways. First, existing shapes can be analyzed similar to our methodology to predict the level of difficulty they pose for replication. Second, the results can be used to design shapes that are easy to replicate, e.g. for gestural interaction. E.g. we expect that a shape that is easy to replicate has compact form (similar to shape 1 and 3), consists of long and short straight lines, strongly concave and convex curved lines, and acute concave and convex corners. On the other hand, a shape that is hard to replicate exhibits a more dispersed form (similar to shape 2), consists of long and short smoothly curved lines, and convex and concave obtuse corners.

We did not investigate longer-term learning in this work. We also have to assume that these results are only true for the family of shapes used here. In further studies we will check if the results can be generalized to other shapes. We also plan to investigate how the orientation or overall sequence of elements affects user performance.

\section{ACKNOWLEDGMENTS}

We thank Dimitrios Gkouskos, Sarathkumar Neelakannan, and Saranya Subramaniam for help in collecting these data.

\section{REFERENCES}

[1] Sutherland, I.E. 1963. Sketchpad, a man-machine graphical communication system.

[2] Holz, C. and Baudisch, P. 2011. Understanding touch. CHI 2011 (Vancouver, BC, Canada, 2011), 2501-2510.

[3] Appert, C. and Zhai, S. 2009. Using strokes as command shortcuts: cognitive benefits and toolkit support. CHI 2009 (2009), 2289-2298.

[4] Meyer, S., Cohen, O. and Nilsen, E. 1994. Device comparisons for goal-directed drawing tasks. Conference companion on Human factors in computing systems - CHI '94 (New York, New York, USA, 1994), 251-252.

[5] Costa, L. da F. and Cesar-Jr., R.M. 2001. Shape Analysis and Classification: Theory and Practice. CRC Press.

[6] Attneave, F. and Arnoult, M.D. 1956. The quantitative study of shape and pattern perception. Psychological Bulletin. 53, 6 (1956), 452-471.

[7] Norman, D.A. and Draper, S.W. 1986. User centered system design: new perspectives on human-computer interaction. L. Erlbaum Associates.

[8] Pinker, S. 2005. So How Does the Mind Work? Mind and Language. 20, 1 (Feb. 2005), 1-24.

[9] Sun, M., Ren, X. and Cao, X. 2010. Effects of Multimodal Error Feedback on Human Performance in Steering Tasks. Journal of Information Processing. 18, (2010), 284-292.

[10] Zabramski, S., Gkouskos, D. and Lind, M. 2011. A comparative evaluation of mouse, stylus and finger input in shape tracing. Proceedings of the 1st European Workshop on HCI Design and Evaluation The influence of domain on Human Computer Interaction design and evaluation (2011), 57-61.

[11] Zabramski, S. 2011. Careless touch: A comparative evaluation of mouse, pen- and touch-input in shape tracing task. OZCHI 2011 (Canberra, Australia, 2011), 329-332.

[12] Attneave, F. 1954. Some informational aspects of visual perception. Psychological review. 61, 3 (May. 1954), 18393.

[13] Pastel, R.L. 2006. Measuring the Difficulty of Steering Through Corners. CHI 2006 (Montréal, Québec, Canada, 2006), 1087-1096.

[14] Holz, C. and Baudisch, P. 2010. The generalized perceived input point model and how to double touch accuracy by extracting fingerprints. Proceedings of the 28th international conference on Human factors in computing systems - CHI '10 (New York, New York, USA, 2010), 581. 